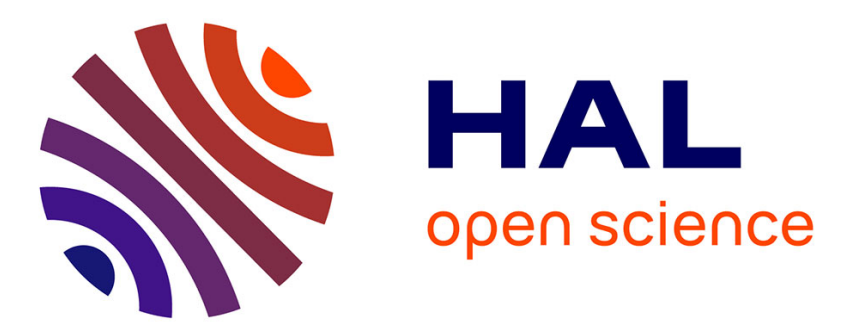

\title{
Putative implication of alpha-amylase loop 7 in the mechanism of substrate binding and reaction products release.
}

\author{
Gwenaëlle André-Leroux, V Tran
}

\section{To cite this version:}

Gwenaëlle André-Leroux, V Tran. Putative implication of alpha-amylase loop 7 in the mechanism of substrate binding and reaction products release.. Biopolymers, 2004, 75 (2), pp.95-108. 10.1002/bip.20096 . hal-02681931

\section{HAL Id: hal-02681931 https://hal.inrae.fr/hal-02681931}

Submitted on 1 Jun 2020

HAL is a multi-disciplinary open access archive for the deposit and dissemination of scientific research documents, whether they are published or not. The documents may come from teaching and research institutions in France or abroad, or from public or private research centers.
L'archive ouverte pluridisciplinaire HAL, est destinée au dépôt et à la diffusion de documents scientifiques de niveau recherche, publiés ou non, émanant des établissements d'enseignement et de recherche français ou étrangers, des laboratoires publics ou privés. 


\author{
G. André 1 \\ V. $\operatorname{Tran}^{2}$ \\ ${ }^{1}$ Institut Pasteur, \\ Unité de Biochimie \\ Structurale, \\ 25 rue du Dr Roux, \\ 75724 Paris Cedex 15, \\ France \\ 2 Unité de Biotechnologie, \\ Biocatalyse et Biorégulation, \\ Faculté des Sciences et \\ techniques 2, \\ rue de la Houssinière, \\ BP 92208, \\ 44322 Nantes Cedex 03, \\ France \\ Putative Implication of $\alpha$ - \\ Amylase Loop 7 in the \\ Mechanism of Substrate \\ Binding and Reaction \\ Products Release \\ Received 2 April 2002; \\ accepted 5 April 2004 \\ Published online 21 July 2004 in Wiley InterScience (www.interscience.wiley.com). DOI 10.1002/bip.20096
}

\begin{abstract}
Amylases are widespread endo-enzymes involved in the hydrolysis of internal $\alpha-(1,4)$ glycosidic linkages of starch polymers. Molecular modeling of amylose-amylase interactions is a step toward enzymatic mechanism understanding and rational design of new enzymes. From the crystallographic complex of barley $\alpha$-amylase AMY2-acarbose, the static aspects of amyloseamylase docking have been characterized with a model of maltododecaose (DP12) (G. André, A. Buléon, R. Haser, and V. Tran, Biopolymers 1999, Vol. 50, pp. 751-762; G. André and V. Tran, Special Publication no. 246 1999, The Royal Society of Chemistry, H. J. Gilbert, G. J. Davies, B. Henrissat, and B. Svensson, Eds., Cambridge, pp. 165-174). These studies, consistent with the experimental subsite mapping (K. Bak-Jensen, G. André, V. Tran, and B. Svensson, Journal of Biological Chemistry, to be published), propose a propagation scheme for an amylose chain in the active cleft of AMY2. The topographical overview of $\alpha$-amylases identified loop 7 as a conserved segment flanking the active site. Since some crystallographic experiments suspected its high flexibility, its putative motion was explored through a robotic scheme, an alternate route to dynamics simulations that consume CPU time. The present article describes the characteristics of the flexibility of loop 7: location and motion in AMY2. A back-and-forth motion with a large amplitude of more than $0.6 \mathrm{~nm}$ was evaluated. This movement could be triggered by two hinge residues. It results in the loop flipping over the active site to enhance the docking of the native helical substrate through specific interactions, it positions the catalytic residues, it distorts the substrate towards its transition state geometry, and finally monitors the release of the products after hydrolysis. The residues involved in the process are now rational mutation points in the hands of molecular biologists. (c) 2004 Wiley Periodicals, Inc. Biopolymers 75: 95-108, 2004
\end{abstract}

Correspondence to: G. André; email: gandre@ pasteur.fr

Biopolymers, Vol. 75, 95-108 (2004)

(c) 2004 Wiley Periodicals, Inc. 
Keywords: molecular modeling; barley $\alpha$-amylase; loop motion; substrate distortion; robotic calculations; hinge residues

\section{INTRODUCTION}

Most proteins display a multiple domain folding. Large and relative motions of domains are often responsible for protein flexibility, and more generally, for protein function. Indeed, domain motions have been shown as essential for a variety of protein functions including catalysis, regulation of activity, transport of metabolites, formation of protein assemblies, and cellular locomotion. ${ }^{1-3}$ Domains often close around the binding site through ligand-induced conformational transition. ${ }^{4}$ Generally, the binding of substrates stabilizes a protein structure in its "closed" conformation, and conversely, the absence of substrate favors the protein structure in its "open" state. ${ }^{1}$ Until now, most experimental information on the mechanism of domain motions has come from x-ray diffraction that resolved opened and/or closed domain structures of proteins. The role of a domain closure during catalysis is often to discard useless water molecules, to position the catalytic residues in their attacking geometry, ${ }^{5}$ and to distort the substrate towards a transition state conformation. ${ }^{1,4}$

Gerstein et al. have studied ${ }^{1}$ the partial mobility of different domains in proteins. From a survey of all the experimentally observed domain movements in proteins, they have defined two low-energy conformational changes: hinge and shear motions. Particularly, among the studied domain movements, hinge motion has been clearly shown for the triose phosphate isomerase $^{6}$ (TIM), the tryptophan synthase $\alpha 2 \beta 2,{ }^{4,7}$ and the inosine $5^{\prime}$-monophosphate dehydrogenase ${ }^{8}$ where these enzymes show a typical $\Omega$ loop,, 910 connecting one $\beta$-sheet to an $\alpha$-helix of the typical TIM barrel folding. In the first two cases, a weak electron density in the region of loop 6 (because located between strand 6 and helix 6 of the $\alpha / \beta$ barrel) combined with fairly high temperature factors, when the proteins are in their native state, implies a high degree of conformational mobility for this loop. In the latter case, loop 6 is clearly visible in the electron density map and is in a different conformation from those in the substrate and substrate analogue-bound structures. It is worth mentioning that $\Omega$ loops are known to be often involved in protein function, folding, and stability, as well as in molecular recognition. ${ }^{10}$ In the case of the TIM enzyme, loop 6 has been suspected to move as a rigid lid thanks to two hinges. Its motion helps to cover the active site and to reinforce the binding of the substrate. ${ }^{2,6}$ In the tryptophan synthase $\alpha 2 \beta 2$ enzyme, closure of loop 6 isolates the active site of the $\alpha$-subunit from solvent and results in interaction between specific and identified residues. ${ }^{4,7}$ In both cases, site-directed mutations confirmed the assumptions and identified residues of the loop as essential for the catalytic activity. Finally, in the inosine $5^{\prime}$-monophosphate dehydrogenase ${ }^{8}$ case, it has been shown that loop 6 follows a similar pattern of hingebody motion and indicates that the protein may be using this loop to bind and sequester substrate and to recruit an essential catalytic residue.

The main interesting and connecting point with TIM isomerase, tryptophan synthase $\alpha 2 \beta 2$, and inosine $5^{\prime}$-monophosphate dehydrogenase is that $\alpha$-amylases exhibits the same $(\alpha / \beta)_{8}$ barrel topology with a similar loop despite completely different enzymatic functions. X-ray diffraction studies have been performed on the wide area of $\alpha$-amylase origins such as Aspergillus oryzae, ${ }^{11,12}$ Aspergillus niger,${ }^{13}$ Bacillus licheniformis, ${ }^{14}$ Bacillus subtilis, ${ }^{15}$ Alteromona haloplanctis, ${ }^{16}$ yellow meal worm, ${ }^{17}$ porcine $^{18,19}$ and human pancreas, ${ }^{20}$ human saliva, ${ }^{21,22}$ and barley. ${ }^{23,24}$ Their native or complex state have revealed experimental evidence for a highly conserved and flexible loop flanking the active site. The loop is suspected to flip over the active site toward a "closed" thus inaccessible position. In the case of PPA, porcine pancreatic $\alpha$-amylase, this loop has been seen in two positions, "open" and "closed" in the crystal, depending respectively on the presence or absence of the competitive inhibitor acarbose. In particular, a significant displacement of $0.5 \mathrm{~nm}$ has been measured for $\mathbf{H}_{\mathbf{P P A}} \mathbf{3 0 5}$ of the loop. ${ }^{18,19,25}$ Temperature factors, particularly high in this protein region, experimentally corroborate such flexibility. The loop, numbered loop 7 in the $(\alpha / \beta)_{8}$ barrel, could trap the substrate by bringing the catalytic aspartic acid $\mathbf{D}_{\mathbf{P P A}} \mathbf{3 0 0}$ to an attacking position facing the other catalytic residues $\mathbf{D}_{\mathbf{P P A}} 197$ and $\mathbf{E}_{\mathbf{P P A}} \mathbf{2 3 3}$. Interestingly, a recent crystallographic article, on human salivary $\alpha$-amylase, probes sensible flexibility and evidences a role in substrate binding and enzyme activity for a short part of this loop 7, $\mathbf{G}_{\mathbf{H S A}} \mathbf{3 0 4}-\mathbf{H}_{\mathbf{H S A}} \mathbf{3 1 0}^{26}$ (PPA numbering and sequence similar in this part of the protein). A similar motion could also be hypothesized for barley loop 7 for $\mathbf{H}_{\mathbf{P P A}} \mathbf{3 0 5}$ has its $\mathbf{H}_{\mathbf{A M Y 2}} \mathbf{2 9 5}$ equivalent. The crystallographic data concerning barley AMY2, either in its native or complex state, does not display an obvious "closed" or "open" position. However, a previous work ${ }^{27}$ showed a very good superimposition 
between oxygens of the crystallographic water molecules and oxygens (glycosidic or hydroxylic) of the modeled substrate. The water oxygens could mimic the substrate oxygens by sharing the same hydrogenbond network, and thus could stick the loop in a closed position. ${ }^{27}$

The present article describes the characterization of this loop: identification, location, conservation, and especially motion. A careful examination of several $\alpha$-amylases has listed and characterized all the structural elements. The structural overview, combined with experimental evidence, identified loop 7 as flexible, part of the active cleft, and particularly conserved among $\alpha$-amylases. Then a thorough exploration of its putative motion was necessary to highlight its dynamic role in the catalytic mechanism. The intrinsic motion and the structural consequences have been explored through a specific strategy called "robotics" where the suspected motion has been deliberately filtered and decomposed into a series of small displacements bordered by to-be-defined "open" and "closed" conformations. It corresponds to a peculiar constraint dynamics protocol. Since previous studies identified key residues of the active cleft for barley AMY2, ${ }^{27,28}$ this methodology has been first applied to this enzyme. This work answers several questions, such as the following: What is the loop motion, its amplitude and its role? What are the consequences on catalysis: residues implied, trapping of transition state, or intermediate structures? Finally, what about a concerted motion?

\section{METHOD}

\section{Loop 7: Identification, Conservation, and Flexibility}

Identification Mode. TAKA, AMY2, and PPA $\alpha$-amylases have been chosen to respectively represent fungal, cereal, and mammal kingdoms. The superimposition mode is based on the backbone overlay of the strictly conserved catalytic triad composed of two aspartic acids and one glutamic acid. The architecture of the catalytic site is known to be perfectly superimposed $^{29}$ (Figure 1). To list all the flexible motifs that might be interesting in a dynamical approach of the catalytic mechanism, the following set of criteria has been defined. All have to be rigorously satisfied: (1) a functional criterion, (2) a conservation criterion, and (3) a structural criterion.

The catalytic efficiency is expected to concern residues located in the active site, so the first criterion requires a location of the motif in the vicinity of the catalytic triad. The second criterion requires for the motif the presence of some consensus sequence region or highly conserved residues among $\alpha$-amylases. The third criterion, by strictly imposing a nonstructural segment, discards automatically $\alpha$-helix or $\beta$-sheet described as rigid parts of a protein. Only loops satisfy this condition. To sum up, the identification of a putative catalytic flexible segment requires it to be a loop fragment, part of the active cleft, with both a primary sequence and a topography conserved within the family 13 of enzymes. According to these criteria, the loop 7 is the only one selected (Figure 1).

Protocol: Definition of two Barycenters and Flexibility Exploration of the Loop Without and With Substrates in the Active Site. The calculations are performed on barley $\alpha$-amylase. The starting data are the crystallographic coordinates of the barley AMY2 complex (1bg9.pdb) with the pseudo-acarbose at 0.28 $\mathrm{nm}$ resolution. ${ }^{23,24}$ Since neither a substrate nor an inhibitor likely to limit or dodge the intrinsic motion of the loop is tolerated in the first stage, the acarbose inhibitor is removed from the active site. So in the first stage, the loop motion is explored without any substrate. In the second stage, to validate the amplitude of motion of the loop and explore its consequences on the hydrolytic mechanism, the motion of the loop is explored in the presence of a real substrate docked in the active site. Since molecular modeling is unable to simulate the cleavage of a covalent bond (only the quantum mechanics can theoretically answer this question and by now only quantum mechanics/ molecular mechanics coupling has approached a solution to this question ${ }^{30}$ ), the two main steps of the catalytic event, besides the proton transfer, are deliberately chosen: the reaction steps before and after the cleavage. For the stage before the hydrolysis, a previously modeled DP5 oligosaccharide ${ }^{31}$ was chosen. Namely, the maltopentaose substrate $\mathbf{A}^{\mathbf{- 1}}-\mathbf{A}-\mathbf{B}-\mathbf{C}-\mathbf{D}$ is composed of glucose rings $\mathbf{A}^{-\mathbf{1}}, \mathbf{A}, \mathbf{B}, \mathbf{C}$, and $\mathbf{D}$ linked with a $\alpha-(1,4)$ bond from the nonreducing to the reducing end. It spans the active site from subsite -2 to subsite +3 . For the stage after the hydrolysis, the loop underwent the same "robotics" strategy of motion in presence of maltose and maltotriose, products resulting from the maltopentaose cleavage. The maltose $\mathbf{A}^{-\mathbf{1}} \mathbf{- A}$ and maltotriose $\mathbf{B}-\mathbf{C}-\mathbf{D}$ occupy respectively the subsites $[-2 ;-1]$ and $[+1 ;+3]$.

In all cases, for sake of CPU time, the enzyme is cut off before any calculations. The protein is restricted to the residues belonging to the loop, to its environment, to the subsites $[-2 ; 3]$, plus the ones necessary for the structural integrity of the enzyme. All residues useless for calculations are discarded. 


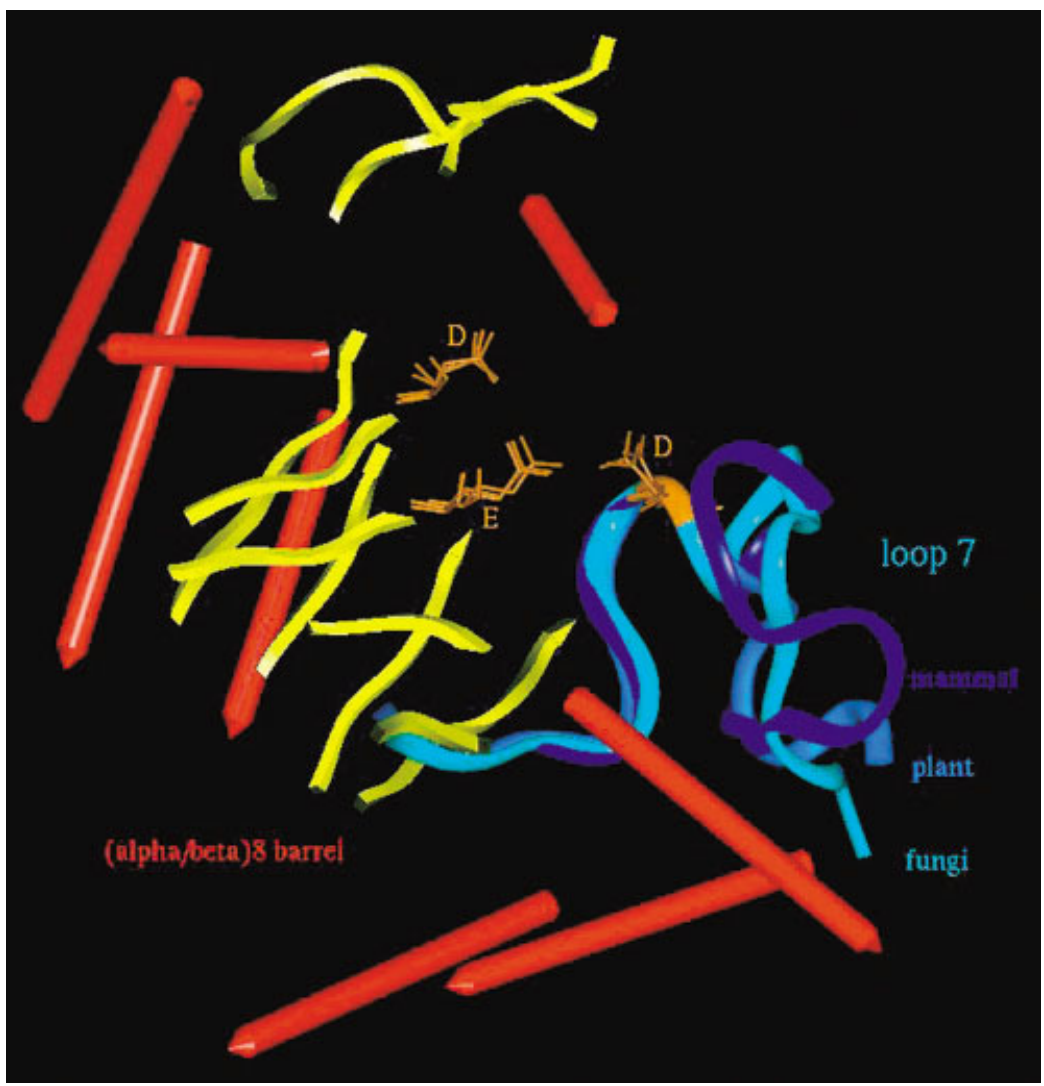

FIGURE 1 Superimposition of TAKA (fungal), AMY2 (cereal), and PPA (mammal) $\alpha$-amylases, based on the backbone overlay of the strictly conserved catalytic triad.

The molecular modeling is carried out on Silicon Graphics computers with the Accelrys packages (San Diego, CA, USA). Molecular displays and energy minimization are performed with Insight II, Biopolymer, and Discover modules. For all calculations, the CFF91 force field is selected.

Internal motions can be simulated by molecular dynamics calculations. Since loop motions are expected to occur in the millisecond time scale, the required dynamics trajectory would have to be sampled in a period of time several times longer than a nanosecond. Moreover, such a long dynamics simulation would guarantee neither a complete description of the loop motion nor the evaluation of its amplitude. Actually, the suspected motion is one of medium frequency and could thus be delicate to extract from the high frequency multiple motions of a dynamic trajectory. Mouawad and Perahia have developed a method based on normal mode analysis. ${ }^{32,33}$ By coupling vibration normal modes and Cartesian coordinates, low frequency motions were identified and characterized. Similarly, a method combining normal mode analysis and molecular dynamics simulation has been set up to analyze domain motions. ${ }^{34}$ These meth- ods are very useful but they are limited to small proteins or to large domain motions.

Due to the environment of this loop 7, the essential movement is a monodirectional back-and-forth motion with a peculiar shifting of the catalytic residue $\mathbf{D}_{\text {AMY2 }} 289$ taking it closer to or further from its catalytic counterparts $\mathbf{D}_{\text {AMY2 }} 179$ and $\mathbf{E}_{\text {AMY2 }} 204$ (Figure 2). Because the movement is decomposed into tiny steps supposed to be a relevant description of the reaction path, the procedure has been qualified as "robotics." (Since it is an original description of the phenomenon, set up for this study, the quotations will be kept throughout the article.) In terms of calculations, the system is partitioned between rigid and mobile segments, where most of the shifting is supported by the loop left free to move. The constraints are thus supported by the remaining enzyme backbone beside the loop as well as the ends of the loop, which are residues $\mathbf{V}_{\mathbf{A M Y} 2} \mathbf{2 8 2}$ and $\mathbf{S}_{\mathbf{A M Y} 2} \mathbf{3 0 1}$ kept backbone fixed. This has been made possible with the definition of two barycenters (Figure 2):

1. B represents the rigid part and corresponds to the center of the mass between the catalytic $D_{\text {AMY2 }} 179$ and $E_{\text {AMY2 }} 204$ backbone atoms. 


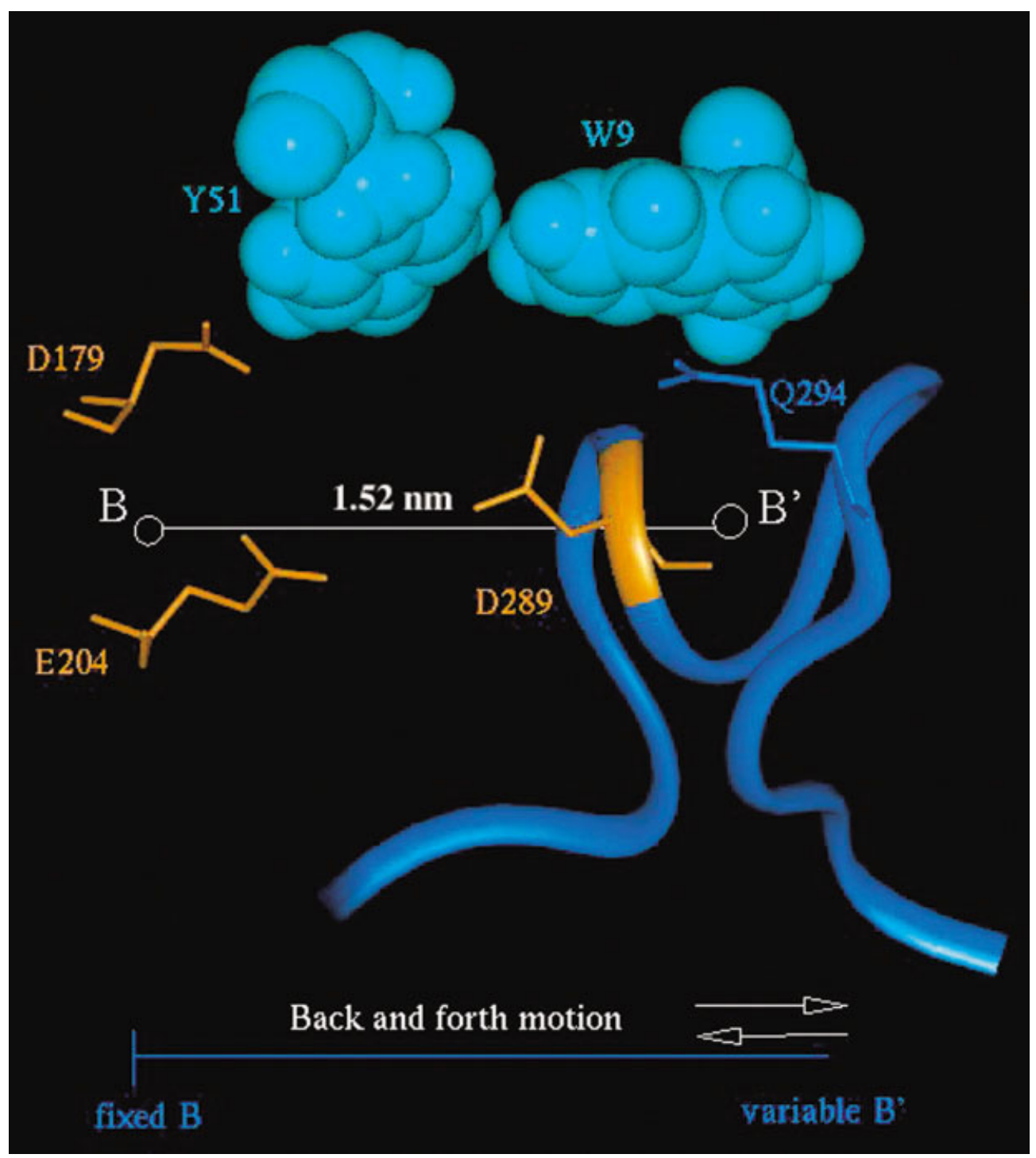

FIGURE 2 Definition of the barycenters $\mathbf{B}$ and $\mathbf{B}^{\prime}$ and description of the robotics strategy.

2. $\mathbf{B}^{\prime}$ represents the mobile loop and is defined from residues $\mathbf{V}_{\text {AMY2 }} 282$ to $\mathbf{W}_{\text {AMY2 }} 297$.

To give a rather continuous description of the phenomenon, the so-called "robotics" calculation is a succession of small displacements of $\mathbf{B}^{\prime}$ toward $\mathbf{B}$ (and backward) under a distance constraint. Every distant restraint is followed by a significant 1500 iteration minimization to relax the internal coordinates of the protein. The goal of the procedure is to decrease the risk of false minima likely to occur with such a constraint trajectory essentially by relaxing the side-chain motion. Starting from the crystallographic position of the loop where the distance between $\mathbf{B}-\mathbf{B}^{\prime}$ is $1.52 \mathrm{~nm}$, two trajectories are needed to describe the conformational space of the loop during its motion.
The two trajectories move from the x-ray position, set up arbitrarily to the medium position. The first one describes the loop flipping over the active site and the second one describes the loop flipping back to a totally accessible crevice. The two trajectories are then merged into a single one where the loop moves back and forth. The resulting trajectory is a succession of displacements of $\mathbf{B}^{\prime}$ toward or away from $\mathbf{B}$ by steps of $0.02 \mathrm{~nm}$ on a range of more than $0.8 \mathrm{~nm}$ :

1. The first trajectory describes a shortening in the [B-B'] distance and brings $\mathbf{D}_{\mathbf{A M Y} \mathbf{2}} \mathbf{2 8 9}$ closer to both $\mathbf{E}_{\mathrm{AMY} 2} 204$ and $\mathbf{D}_{\mathrm{AMY} 2} \mathbf{1 7 9}$ step by step. This motion narrows the catalytic cleft and finally leads to a completely closed position for loop 7. This artificial position is particularly 
much closer than the experimental $\mathrm{x}$-ray conformation that refers to the complex conformation known to trap substrates or inhibitors.

2. The second trajectory corresponds to the reverse motion that enlarges the active cleft from the medium x-ray position to the "open" one. It corresponds to an increase in the $\mathbf{B}-\mathbf{B}^{\prime}$ ' distance, with $\mathbf{D}_{\mathrm{AMY} 2} \mathbf{2 8 9}$ going further to the barycenters $\left(\mathbf{E}_{\text {AMY2 }} 204 ; \mathbf{D}_{\text {AMY2 }}\right.$ 179). The broadening has been fully explored so that the loop ends up in a completely open position.

The distance of $1.52 \mathrm{~nm}$ is artificially set to the relative value zero and the displacements towards the "closed" position are qualified with negative distance values; conversely the displacements towards the "open" position are noted as positive distance values (Figure 2). The experimental position, refined with a minimization similar to any step of the robotics strategy except the $\mathbf{B}-\mathbf{B}^{\prime}$ distance constraint, is obviously an acceptable geometry, so that its energy value can be taken as an energy threshold. Such a criterion borders the loop motion as it encompasses realistic conformations and discards odd ones at each extremity. Any conformation beyond this energetic threshold is geometrically and energetically unbearable for the protein.

As mentioned above, the validation of the loop motion as well as the comprehension of the different steps of the catalytic event imply the knowledge of the substrate behavior during the loop motion. For those reasons, a similar exploration is performed in the presence of a real substrate in the active site. Starting from the previously modeled maltopentaose ${ }^{27}$ in the active site of barley $\alpha$-amylase with the loop this time in an "open" position, the "robotics" explores the motion of the loop until it reaches the crystallographic position supposed to be the very last step before the protonation of the catatytic bond. For a chronological continuity, the substrate is then artificially cleaved into maltose and maltotriose products known to occur from the hydrolysis of a maltopentaose and the loop is then moved forward until it reaches a "closed" position.

\section{RESULTS AND DISCUSSION}

\section{Characterization of Loop 7}

Identification of Loop on the Set of Functional and Biochemical Criteria. This superimposition displays a close overlay of the structural elements of the $(\alpha / \beta)_{8}$ barrel where the eight helices and eight sheets have respectively their helical and strand equivalents. Only loop 7, located between helix A- $\alpha 7$ and sheet A- $\beta 7$ of the $(\alpha / \beta)_{8}$ barrel, satisfies the three criteria previously defined. Experimental data strongly support the flexibility of this loop 7 that flanks the active side and contains the fifth consensus region in $\alpha$-amylases: F-V-D-N-H-D. The last residue of this sequence is the aspartic residue of the catalytic triad where the following numberings $\mathbf{D}_{\text {TAKA }} \mathbf{2 9 8}, \mathbf{D}_{\text {AMY2 }} 289$, and $\mathbf{D}_{\mathbf{P P A}} \mathbf{3 0 0}$ refer respectively to TAKA, AMY2, and PPA $\alpha$-amylases. It is worth mentioning that the three loops 7 show a backbone superimposition that goes largely beyond the consensus sequence and that gives a particularly low average RMS of $0.017 \mathrm{~nm}$ (Figure 1).

Main Structural Features of Loop 7. Despite a homologous folding for the part of the loop that corresponds also to the consensus sequence, the length of loop 7 varies with the $\alpha$-amylase origin. The TAKA amylase loop 7 has 10 amino acid residues ( $\mathbf{F}_{\text {TAKA }}$ 292-F $\left._{\text {TAKA }} \mathbf{3 0 1}\right)$, AMY2 has 15 residues $\left(\mathbf{F}_{\text {AMY2 }} \mathbf{2 8 2 - P _ { \text { AMY2 } }} \mathbf{3 0 0}\right)$, and PPA 20 residues $\left(\mathbf{F}_{\text {PPA }} \mathbf{2 9 5}-\mathbf{F}_{\text {PPA }} \mathbf{3 1 5}\right)$. More generally, fungal and bacterial $\alpha$-amylases display a particularly short loop with an average of 15 residues while cereal and mammal $\alpha$-amylases show a longer loop ranging from 15 to 25 residues.

Interestingly, some structural features provide significant internal rigidity to the mobile system. The loop joining sheet $\beta 7$ and helix $\alpha 7$ has a typical $\Omega$ loop folding. ${ }^{9,10,35}$

1. The $\Omega$ loops are $\Omega$-shaped nonstructural segments, located on the external side of globular proteins with a planar backbone. ${ }^{36}$ They are characterized by a lack of repetitive dihedral angles and of regular hydrogen bonds as well. However, intraloop hydrogen bonds and other elements such as turn are required to assure some rigidity to the loop and thus to guarantee its structural integrity during the motion. In cereal and mammal amylases, loop 7 adopts a typical $\Omega$ shape with a double turn at its top. Residues $\mathbf{N}_{\mathrm{AMY} 2} \mathbf{2 8 7}-\mathbf{G}_{\mathrm{AMY} 2} \mathbf{2 9 1}$ or $\mathbf{N}_{\mathrm{PPA}} \mathbf{2 9 8 -}$ $\mathbf{Q}_{\mathbf{P P A}} \mathbf{3 0 2}$ form the first turn that Kadziola qualified, in barley, as $\alpha 7 \mathrm{a}^{23}$ The absence of such a turn, in the fungal amylase, is counterbalanced by a short twin loop parallel to loop 7 .

2. Residue $\mathbf{S}_{\text {AMY2 }} 292$, located in the double turn, shows $(\varphi, \psi)$ values located in the forbidden region of the Ramachandran plot. It thus limits drastically the motion of both this residue and its neighbors.

3. Finally, the internal hydrogen-bond network for the three loops is dense and also participates in 
Table I Internal Hydrogen Bonds of Loop 7 in AMY2 in the Crystallographic Position

\begin{tabular}{llc}
\hline Atom 1 & Atom 2 & Distance (nm) \\
\hline D286 OD1 & F299 N & 0.30 \\
N287 OD1 & T290 N & 0.29 \\
N287 OD1 & D289 N & 0.28 \\
N287 N & T290 OG1 & 0.28 \\
N287 O & G291 N & 0.28 \\
H288 O & Q294 N & 0.28 \\
D289 O & M296 N & 0.29 \\
T290 OG1 & G291 N & 0.30 \\
T290 O & W297 N & 0.29 \\
G291 O & H295 N & 0.29 \\
S292 OG & T293 N & 0.28 \\
P300 O & R303 N & 0.30 \\
D302 N & D302 OD2 & 0.29 \\
\hline
\end{tabular}

maintaining the general architecture of the loop. Tables I-VI list the intra- and interhydrogen bonds of AMY2 loop for the medium and extreme positions. The first three tables concern the intraloop hydrogen bonds from $\mathbf{F}_{\text {AMY2 }} 284$ to $\mathbf{D}_{\text {AMY2 }} \mathbf{3 0 2}$, the last three tables register the interhydrogen bonds between loop 7 and its environment. In those tables, the residues involved will be seen as essential in the loop mobility despite a low hydrogen-bond number.

The alternative solution of a poor hydrogen-bond network could be used in the case of a loop for which the folding flexibility would be particularly required. In the case of loop 7 , the forbidden $(\varphi, \psi)$ values of S292, the tight hydrogen-bond network combined to a $\Omega$-shape folding greatly favor the suspected rigidbody-type motion. This constrained folding is also consistent with a hinge-type motion where the hinge residues could be located at the bottom of the loop.

Table II Internal Hydrogen Bonds of Loop 7 in AMY2 in the "Closed" Position

\begin{tabular}{llc}
\hline Atom 1 & Atom 2 & Distance (nm) \\
\hline D286 OD2 & T290 OG1 & 0.32 \\
N287 OD1 & T290 HN & 0.30 \\
N287 O & G291 N & 0.28 \\
H288 O & Q294 NE2 & 0.30 \\
D289 OD1 & Q294 NE2 & 0.30 \\
W297 N & T290 O & 0.29 \\
G291 O & Q294 N & 0.29 \\
D302 OD1 & R303 NH1 & 0.29 \\
D302 OD2 & R303 NE & 0.28 \\
\hline
\end{tabular}

Table III Internal Hydrogen Bonds of Loop 7 in AMY2 in the "Open" Position

\begin{tabular}{llc}
\hline Atom 1 & Atom 2 & Distance (nm) \\
\hline N287 N & T290 OG1 & 0.30 \\
Q294 NE2 & D289 OD1 & 0.30 \\
H295 NE2 & S292 O & 0.30 \\
R303 N & P300 O & 0.29 \\
V304 N & S301 O & 0.24 \\
R303 NH1 & D302 OD1 & 0.29 \\
R303 NE & D302 OD2 & 0.28 \\
\hline
\end{tabular}

Environment of Loop 7. An accurate exploration of the environment around loop 7 shows the presence of highly conserved aromatic residues: $\mathbf{Y}_{\mathbf{A M Y 2}} \mathbf{5 1}$ and $\mathbf{W}_{\text {AMY2 }}$ 9, $\mathbf{Y}_{\text {PPA }} 62$ and $\mathbf{W}_{\text {PPA }} 58$, and $Y_{\text {TAKA }} 82$. TAKA-amylase lacks an equivalent $\mathbf{W}_{\text {AMY2 }}{ }^{9}$ or $\mathbf{W}_{\text {PPA }} 58$ residue. A detailed spotting of AMY2 identifies residues creating a hydrophobic upper rail on which leans the polar residue $\mathbf{Q}_{\mathrm{AMY2}} 294$. $\mathbf{W}_{\mathbf{A M Y 2}} 9$ is actually sandwiched between $\mathbf{Q}_{\mathbf{A M Y 2}} \mathbf{2 9 4}$ of loop 7 and $\mathbf{Q}_{\mathbf{A M Y 2}} \mathbf{4 9}$ so that its side chain is fixed in a given conformation. This rail feature combined with the $\Omega$-shaped loop is fully coherent with the back and forth motion because $\mathbf{Q}_{\mathrm{AMY} 2} \mathbf{2 9 4}$ could slide along the hydrophobic patch $\mathbf{W}_{\text {AMY2 }}$ 9. $\mathbf{Q}_{\text {AMY2 }} 294$ could act as a monitor to drive the loop forward and to bring D $_{\text {AMY2 }} 289$ closer to its catalytic D $_{\text {AMY2 }} 179$ and $\mathbf{E}_{\mathrm{AMY2}} 204$ counterparts (Figure 1).

A final structural element is worth underlining as it could highlight some obscure points in the catalytic mechanism. Crystallographic data of TAKA, PPA, and AMY2 enzymes give water oxygens. Highly conserved oxygens have been shown to be located in a water pocket or channel adjacent to the active site. One wall of this water trap is formed with the conserved sequence F-V-D-N-H-D of loop 7. Kadziola suggested, in the case of AMY2, that the extreme water molecule of the channel ( $\left.\mathbf{W}_{\mathbf{A M Y 2}} \mathbf{6 0 7}\right)$, hydrogen bridged between $\mathbf{E}_{\mathbf{A M Y 2}} 204$ and $\mathbf{D}_{\text {AMY2 }} 289$, could act as the nucleophile during hydrolysis. The

Table IV Hydrogen-Bond Network Between Loop 7 in the Crystallographic Position and Surroundings in AMY2

\begin{tabular}{llc}
\hline Atom 1 & Atom 2 & Distance (nm) \\
\hline S292 OG & W9 N & 0.30 \\
T293 OG1 & G10 OE2 & 0.28 \\
W297 O & K249 NZ & 0.29 \\
W297 O & N253 ND2 & 0.33 \\
\hline
\end{tabular}


Table V Hydrogen-Bond Network Between Loop 7 in the "Closed" Position and Surroundings in AMY2

\begin{tabular}{llc}
\hline Atom 1 & Atom 2 & Distance (nm) \\
\hline V282 N & T243 O & 0.30 \\
D245 N & V282 O & 0.29 \\
T283 O & C319 N & 0.30 \\
K249 NZ & D286 OD2 & 0.29 \\
Q5 OE1 & H288 N & 0.29 \\
Q5 NE2 & H288 ND1 & 0.27 \\
E204 OE2 & D289 OD2 & 0.29 \\
D179 OD1 & D289 OD2 & 2.9 \\
S292 OG & T293 N & 2.9 \\
\hline
\end{tabular}

loop motion, by implying a sensible motion of $\mathbf{D}_{\mathrm{AMY2}}{ }^{289}$, could trigger off the ejection of W $_{\text {AMY2 }} 607$ towards the glycosidic linkage and thus could drive the water molecule to the attacking position expected in the second step of the reaction mechanism. In that way, the loop motion could not only enhance the turnover of the enzyme but could also favor the water supply of this channel or pocket.

\section{Flexibility of Loop 7.}

Energetic Diagram and Amplitude Motion When the Active Site Is Empty. Molecular mechanics protocol adopted a deliberately filtered "robotics" analysis. The successive variations of $\mathbf{B}^{\prime}$ displacements are considered as discrete snapshots of the loop motion and a sequential description of the reaction pathway. All the positions after minimization are merged into a unique trajectory file where each $\left[\mathbf{B}-\mathbf{B}^{\prime}\right]$ distance corresponds to a total potential energy value. The total potential energy variation versus $\left[\mathbf{B}-\mathbf{B}^{\prime}\right]$ distance is plotted in Figure 3.

The diagram shows a rather symmetrical curve with energy increases for the two extreme positions and interestingly the medium crystallographic one. The energy plot confirms a back-and-forth motion as acceptable. According to the energetic criterion, three positions are discriminated: the crystallographic one (the medium position) and the two extreme positions ("closed" and "open") bordering out the loop displacement, respectively located at $-0.24 \mathrm{~nm}$ and $+0.4 \mathrm{~nm}$ from the crystallographic position; the former value corresponds to a "closed" position, the latter to an "open" one. Despite a rather rough energy criterion, this bordering allows a large amplitude of motion of more than $0.6 \mathrm{~nm}$.

Key Points of the Loop Motion. Generally speaking, the trajectory tracks down the conformational reaction path of the loop during its motion. The extreme and intermediate positions seen in Figure 3 show that the
$\Omega$ shape is kept all along the loop motion, thus confirming the architectural importance of the $\Omega$ loop characteristics previously underlined.

Crystallographic Position. The crystallographic conformation and position of the loop is red in Figure 3. Interestingly, the energy plot displays a peak at this position. As it is a refined experimental conformation, a minimum in potential energy should be expected. Among the different hypotheses that can explain the original shape of the curve, one could argue some "memory effect" of the inhibitor due to the initial coordinates of the complex AMY2/acarbose. Even if the robotics calculations demand that the inhibitor should be discarded, residues that used to be in contact with the inhibitor could have maintained their side chains in specific geometry difficult to relax. The second hypothesis relates also to the acarbose inhibitor that is supposed to mimic the transition state. This suggests that the enzyme is deluded and adopts an activated conformation, thus increasing its conformational energy. In all cases, the loop in this x-ray and intermediate position would initiate the distortion of the substrate towards its transition state.

To elucidate this tricky point, a continuous and reverse loop motion is performed completely from the newly defined "closed" position toward the "open" one. A similar strategy is used: same distance range, same calculation protocol (step of $0.02 \mathrm{~nm}$ for each [B-B'] distance variation, 1500 iteration minimization under similar constraints). This second trajectory no longer displays an energy increase at the corresponding crystallographic position. The energy plot shows a regular curve with maxima at the extreme positions and minimum at the medium crystallographic position. This robotics trajectory confirms once more the possibility of a back-and-forth motion for loop 7. The increase observed in the previous trajectory at the crystallographic position could be due to a kind of memory effect for the protein that used to have its crevice occupied with the acarbose inhibitor.

\section{Extreme Geometries.}

The opened position. The opened position is shown in green in Figure 3. The energy diagram

Table VI Hydrogen-Bond Network Between Loop 7 in the "Open" Position and Surroundings in AMY2

\begin{tabular}{llc}
\hline Atom 1 & Atom 2 & Distance $(\mathrm{nm})$ \\
\hline V282 N & FT243 O & 0.30 \\
D245 N & V282 O & 0.28 \\
C319 N & T283 O & 0.29 \\
K249 OZ2 & V285 O & 0.29 \\
H324 NE2 & D286 OD1 & 0.29 \\
\hline
\end{tabular}




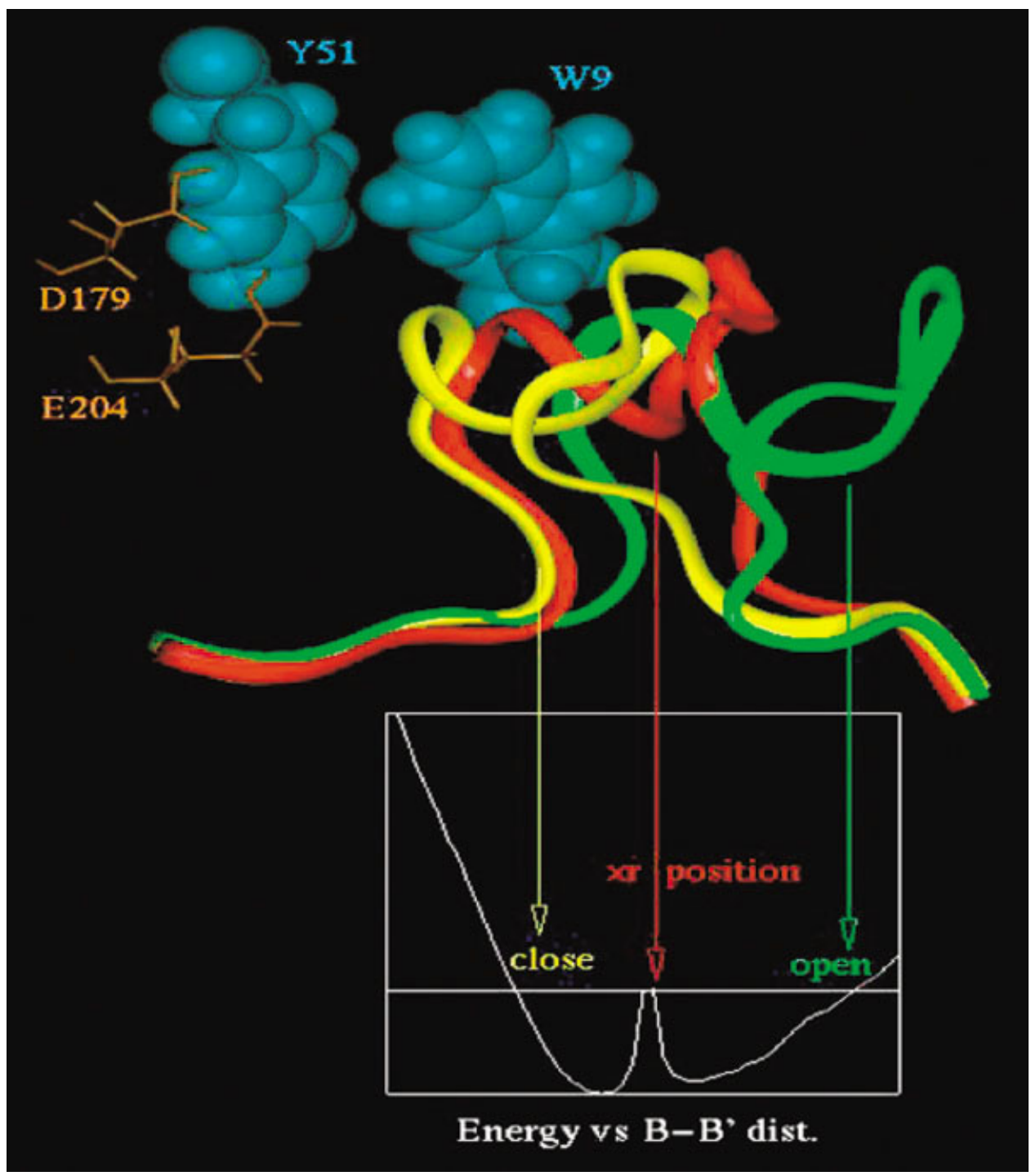

FIGURE 3 Energy diagram (energy plot vs [B-B'] distance) and amplitude motion of the loop along its trajectory. Identification of the open (green), medium (red), and close (yellow) positions.

shows a putative motion of $0.4 \mathrm{~nm}$ from the starting crystallographic position without noticeable steric conflict. Despite no measurement performed in terms of surface accessibility, the catalytic cleft is obviously enlarged. The broadening of the cleft while the loop flips back to an open position could facilitate the arrival of substrate, particularly the helical one. This capability to dock a helical chain of substrate should confirm the efficiency of barley amylases on native solid substrate. These enzymes are actually known to act on starch granules during the physiological conditions of germination where amylose and amylopectin chains are folded in helical conformations. This result could be a step toward the understanding of amylolysis in heterogeneous phase condition.
As the loop flips over the active site, some of its residues could be implied in the early stage of substrate docking. Several residues involved in subsite binding belong to loop 7, especially $\mathbf{H}_{\mathbf{A M Y} 2} \mathbf{2 8 8}$, $D_{\text {AMY2 }} 289$, and $M_{\text {AMY2 }} 296$ that have been identified $^{27,28}$ as critical for the docking of short or for long substrates. Among residues that also define the catalytic crevice, $\mathbf{Y}_{\mathrm{AMY} 2} 104$ and segment $\left[\mathbf{E}_{\mathrm{AMY} 2} 204-\right.$ $\mathbf{K}_{\mathrm{AMY} 2} 2 \mathbf{2 1 6}$ ] from loop 5 could act in synergy with the loop 7 and thus partially disrupt the helical structure of the amylose chain. Through strengthened interactions, the first steps of the substrate docking could be facilitated.

The closed position. The so-called "closed" conformation corresponds to the yellow position in Fig- 
ure 3 . The diagram displays putative motion amplitude of $0.25 \mathrm{~nm}$ between the medium and the close positions. The energy variations during the loop motion is too delicate to interpret qualitatively; however, the large increase in energy, beyond the cutoff, can obviously be explained by steric clashes between residues of the loop within its environment.

We propose the following chronological and catalytic process as the loop moves from the intermediate position to the "closed" one:

First, the dynamical rearrangement of the residues of the crevice results in a tighter binding of the substrate and more generally in a correct positioning of the reacting agents.

Then the negatively charged $\mathbf{D}_{\mathbf{A M Y 2} 2} \mathbf{2 8 9}$ moves closer to the substrate. On the other side of the cleft, the protonated $\mathbf{E}_{\mathbf{A M Y 2}} \mathbf{2 0 4}$, now at a catalytic distance from the reacting sugars, is able to transfer its proton to the glycosidic oxygen, thus promoting the distortion of the glucose ring and the formation of the transition state.

Finally, the loop motion could promote the ejection of a water molecule from the water channel and could place it in an attacking position. This point is particularly interesting as the water molecule could act as the nucleophile in the reaction process.

In a further chronological step while the loop keeps on moving in, some cooperative side-chain repositioning between the loop residues and their environment could favor the reaction product release. This point will be particularly illustrated in the paragraph of the loop motion in the presence of products.

Loop Characteristics in the Presence of Substrate. To validate the loop flexibility, we studied the amplitude of the loop motion and its functional consequences when the active site is filled with substrates. This "robotics" study actually requires two trajectories. The first (part I) describes the maltopentaose behavior when the loop moves from an "open" state to the crystallographic position; then the second trajectory (part II) describes the behavior of the products when the loop moves from the RX position to the "closed" position (Figure 4, upper). The expected result is a functional comprehension of the loop motion. The first trajectory shows how the loop motion acts realistically both for the tight docking and for the distortion of the substrate towards its transition state geometry while the second trajectory displays a dynamic release of the products once the hydrolysis is done.

Loop Motion with the Maltopentaose. During the trajectory, specific frames have been deliberately selected as they describe very specific steps of the reactional path. The images in Figure 4 (lower right) correspond respectively to

- a completely open loop (green loop),

- the half open position and the minimum in energy (light green), and

- the RX position (red loop).

The maltopentaose $\mathbf{A}^{\mathbf{- 1}} \mathbf{- A}-\mathbf{B}-\mathbf{C}-\mathbf{D}$ occupies subsites $\mathbf{- 2}$ to $\mathbf{+ 3}$ so that the glucose ring $\mathbf{A}$ is docked in subsite $\mathbf{- 1}$ where the catalysis occurs. This ring is highly suspected to go through a distorted half-boat conformation during the catalysis. The set of Pucker polar parameters $(Q, \Theta, \varphi)$ characterize the distortion of a glucose conformation where the ${ }^{4} C_{1}$ chair conformation is the reference for a $\alpha$-D glucose ring. Table VII shows the evolution of the Pucker parameters for the glucose ring A during the loop motion. Actually, as the loop moves forward, the substrate gets trapped tighter by stacking interactions between ring $\mathbf{A}$ and $\mathbf{Y 5 1}$ and between ring $\mathbf{C}$ and W206. Then, as the loop goes on moving in, the ring $\mathbf{A}$ in subsite $\mathbf{- 1}$ undergoes a significant distortion measured with the $\Theta$ parameters going from $11.95^{\circ}$ to $23.7^{\circ}$. The distortion, results in a final half-boat $\mathrm{H}_{2}$ conformation for ring $\mathbf{A}$ known to be the transition state conformation. The transition from the ${ }^{4} \mathrm{C}_{1}$ conformation to the $\mathrm{H}_{2}$ conformation is helped by the residues $\mathbf{H 2 8 8}$, D204, and D289 (Figure 4, lower right).

Loop Motion with the Maltotriose and Maltose. During the trajectory, some specific frames are selected as they interestingly illustrate the release of the products during the reactional path. When the loop flips over the active site, numerous specific interactions between the sugar and the protein are disrupted, thus weakening the docking of the substrate. In fact, during the closure of the crevice, residues $\mathbf{Y}_{\text {AMY2 }} 51, \quad \mathbf{H}_{\mathrm{AMY} 2} 288, \quad \mathrm{~W}_{\text {AMY2 }} 206$, $\mathbf{M}_{\text {AMY2 }} 296$, and $\mathbf{W}_{\text {AMY2 }} 297$ accommodate their side chain so as to reduce the steric hindrance. During their repositioning, they unstick respectively from rings $\mathbf{A}, \mathbf{B}, \mathbf{C}$, and $\mathbf{D}$ (Figure 4, lower left: on this figure for sake of clarity, only the unstick of the maltotriose B-C-D is shown). Indeed, some loop residues compete with glucose rings to create stacking interactions with aromatic residues. Actually, two pairs of amino acid residues: H $_{\text {AMY2 }} 288 / \mathbf{Y}_{\text {AMY2 }} 51$ and M $_{\text {AMY2 }} 296 /$ $\mathbf{W}_{\text {AMY2 }} 206$ illustrate this putative property (Figure 4 , lower left and right). The first pair of residues is a highly conserved set. What is more, $\mathbf{Y}_{\mathbf{A M Y 2}} \mathbf{5 1}$ and $\mathbf{W}_{\mathrm{AMY2}} \mathbf{2 0 6}$ are referenced as conserved stacking patches ${ }^{37-39}$ for substrate glucose rings. ${ }^{27,28}$ During the loop closure, $\mathbf{H}_{\mathbf{A M Y} 2} \mathbf{2 8 8}$ residue moves in and 


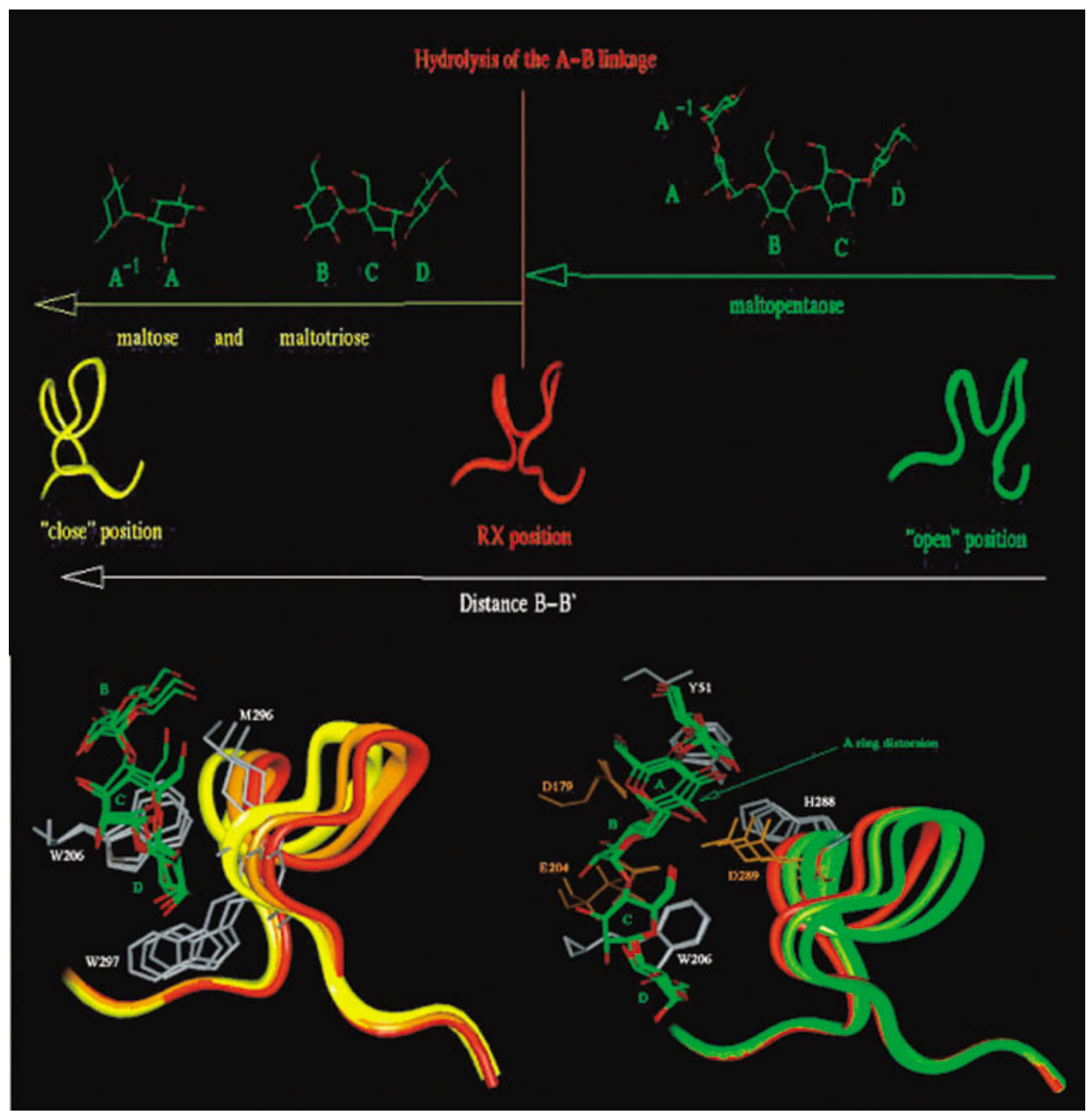

FIGURE 4 Upper: chronological and functional comprehension of the loop motion. Lower right: distortion of the maltopentaose at subsite $\mathbf{- 1}$ to get to its transition state geometry. Lower left: release of the cleaved substrate, details of the leaving maltotriose on the reducing end.

creates a hydrogen bond with the side chain of $\mathbf{Y}_{\mathbf{A M Y 2}} \mathbf{5 1}$, thus inducing a relevant reorientation of the tyrosine side chain. Such competing interaction weakens the affinity between $\mathbf{Y}_{\mathbf{A M Y 2}} \mathbf{5 1}$ and the glucose ring $\mathbf{A}$ and could be responsible for the disruption of the stacking interaction. Similarly, the significant displacement of $\mathbf{M}_{\mathbf{A M Y 2}} 296$ residue initiates a significant displacement of $\mathbf{W}_{\mathbf{A M Y 2}} \mathbf{2 0 6}$ side chain, thus decreasing the binding affinity between glucose ring $\mathrm{C}$ and the stacking subsite $\mathbf{W}_{\text {AMY2 }} 206$.
Hinge Residues. An overview of protein motions showed that the intrinsic flexibility of some segments can be classified into hinge or shear motions. ${ }^{1}$ In our case, the back and forth motion should be obviously compared to the first-type-hinge-motion as seen for the TIM $\Omega$ loop. $^{6-8}$ Despite neither significant nor very located variation in the $\mathrm{C} \alpha$ dihedral values, the $\Omega$ shape of the $(\alpha / \beta)_{8}$ barrel loop 7 combined with a pivotal displacement of the $\mathbf{H}_{\text {AMY2 }} \mathbf{2 8 8}$ and D $_{\text {AMY2 }} 289$ residues are strong structural features 
Table VII Pucker Parameters Evolution for Ring A Docked in Subsite -1

\begin{tabular}{lccccc}
\hline $\begin{array}{l}\text { Loop Position, } \\
\text { Energy }\end{array}$ & $\begin{array}{c}\text { Open, } \\
\text { Maximum }\end{array}$ & $\begin{array}{c}\text { Intermediate, } \\
\text { Intermediate }\end{array}$ & $\begin{array}{c}\text { Half Open, } \\
\text { Minimum }\end{array}$ & $\begin{array}{c}\text { Intermediate, } \\
\text { Intermediate }\end{array}$ & $\begin{array}{c}\mathrm{RX}, \\
\text { Maximum }\end{array}$ \\
\hline$Q$ & 0.542 & 0.541 & 0.542 & 0.542 & 0.542 \\
$\Theta$ & 11.95 & 14.23 & 16.05 & 20.00 & 23.68 \\
$\varphi$ & 74.85 & 75.68 & 77.30 & 83.56 & 89.59 \\
\hline
\end{tabular}

which suggest a hinge-type motion. Indeed, one could expect that residues located at the bottom could act as lever arm residues and a small variation in their $(\varphi, \psi)$ values will induce a significant displacement of residues located at the top. Interestingly, those residues are the catalytic $\mathbf{D}_{\mathbf{A M Y 2} 2} \mathbf{2 8 9}$ and the important $\mathbf{H}_{\mathrm{AMY2}} 288$ and $\mathbf{M}_{\mathrm{AMY} 2} 296$ residues. A thorough exploration identified $\mathbf{V}_{\text {AMY2 }} 285$ and $\mathbf{F}_{\text {AMY2 }} 299$ as putative hinge residues with $\mathbf{V}_{\mathbf{A M Y 2}} \mathbf{2 8 5}$ interestingly belonging to the fifth consensus sequence FVDNHD. The two residues are located at the bottom sides of the $\Omega$ loop where the loop gets narrower. They are both hydrophobic residues connected with van der Waals interaction and with their side chain always in tight contact during the loop motion.

More generally, a comparison between AMY2, TAKA, and PPA $\alpha$-amylases revealed a similar "gear" mechanism with a conserved set of hinge residues located in the same area of the $\Omega$ loop. In TAKA-amylase, the hinge pair is strictly conserved with $\mathbf{V}_{\text {TAKA }} 293$ and $\mathbf{F}_{\text {TAKA }} 301$ residues, in PPA amylase, $\mathbf{V}_{\text {PPA }} \mathbf{2 9 6}$ is strictly conserved while F $_{\text {AMY2 }} 299$ is replaced by a similarly hydrophobic isoleucine $\mathbf{I}_{\mathbf{P P A}} \mathbf{3 1 3}$.

\section{CONCLUSIONS}

This work is a first step toward a structural and functional three-dimensional mapping of $\alpha$-amylases. The comparison of $\alpha$-amylases from different origins combined crystallographic data, consensus sequence superimposition, and structural and functional features identification. Criteria have been set up here to select regions that are interesting as flexible and functional motifs. The criteria require a nonfolded segment (structural criterion) that contains a consensus sequence (biochemical criterion) and that flanks the active site (functional criterion).

From a survey of $\alpha$-amylases and data selected from fungal (TAKA), cereal (AMY2), and mammal (PPA, HSA) amylases, a loop has been identified as potentially flexible and strongly involved in the amylolysis. Loop 7 has been proved here to be the loop among the eight ones of the TIM barrel that satisfies the structural, functional, and biochemical criteria. Among the $\alpha$-amylases, all the loops 7 display a characteristic $\Omega$-shape loop, referenced as flexible in many TIM barrel proteins with different function. This $\Omega$ loop is enriched with a strong internal hydrogen-bond network, with a double turn on top, and in the case of AMY2, with forbidden $(\varphi, \psi)$ values in the Ramachandran plot for a serine residue. It induces some internal rigidity for the loop but this tenseness has been shown to be consistent with the suspected back and forth movement. A detailed exploration of AMY2 loop 7 surroundings shows a rail that could support and monitor the loop in its motion. This rail is formed of other loops of the TIM barrel, generally speaking, most of the loops could deserve a study but the paper here focused on loop 7 .

Barley AMY2 whose crevice was already characterized in terms of subsite residues ${ }^{27,28}$ was chosen to explore the putative catalytic loop 7. An original robotics strategy has been developed where the very high and very low frequency motions were deliberately filtered to get into the loop motion of medium frequency. As a back-and-forth motion was suspected, loop 7 has been successively moved in and out of its crystallographic position considered as an intermediate one. The loop flexibility has been validated with a similar "robotics" strategy in the presence of substrate or products of hydrolysis. In all cases, the calculations explored the mobility of the loop and bordered its motion with a precise identification of "close" and "open" conformations. The loop displacement showed a non-negligible amplitude of more than 0.6 $\mathrm{nm}$; a $0.4 \mathrm{~nm}$ displacement from the "open" to the medium positions and a $0.24 \mathrm{~nm}$ displacement from the medium to the "closed" positions.

From this study, the motion could be seen as a cooperative displacement of residues, initiated by hinge residues located at the bottom of the loop. A small displacement of the pivotal $\mathbf{V}_{\mathbf{A M Y 2}} \mathbf{2 8 5}$ and F $_{\text {AMY2 } 299}$ could trigger the loop movement. Consistently, a similar set of "hinge" residues has been found highly conserved in $\alpha$-amylases. The two res- 
idues are now proposed as rational mutation points to validate or not the hinge hypothesis.

A chronological scheme of the loop motion is proposed with extreme conformations corresponding to key steps in the adsorption/desorption mechanism. The "open" conformation obviously enlarges the cleft so that the adsorption of substrate, even the native helical one, is largely facilitated. When the loop flips over, the residues of the loop such as $\mathbf{H}_{\mathrm{AMY} 2} \mathbf{2 8 8}$, $\mathbf{Q}_{\mathrm{AMY} 2} 294$, and $\mathbf{M}_{\mathrm{AMY} 2} 296$ move forward in synergy with their crevice counterparts, respectively $\mathbf{Y}_{\text {AMY2 }} 51, \mathbf{W}_{\text {AMY2 }} 9$, and $\mathbf{W}_{\text {AMY2 }} 206$ to reinforce the substrate binding, to promote the transition state formation and then to position $\mathbf{D}_{\mathbf{A M Y} 2} \mathbf{2 8 9}$ to an attacking position for catalysis. Located in the close vicinity of the glycosidic linkage and the protonated catalytic $\mathbf{E}_{\mathrm{AMY} 2} 204$ residue, $\mathbf{D}_{\text {AMY2 }} 289$ along with $\mathbf{D}_{\text {AMY2 }} 179$ could stabilize the transition state through a covalent intermediate after the departure of the leaving group. The ultimate closure of the loop considerably decreases the width of the cleft and enhances cooperative side-chain motions of residues $\mathbf{Y}_{\mathbf{A M Y}} \mathbf{5 1 /}$ $\mathrm{H}_{\mathrm{AMY} 2} 288$ and $\mathrm{W}_{\mathrm{AMY} 2} 206 / \mathrm{M}_{\mathrm{AMY} 2} 296$, thus favoring the release of the cleaved fragments. In a final step, desorption of the products is thus largely favored.

In the calculations, the water molecules have been deliberately discarded. It is known that $\alpha$-amylases display a water pocket in the vicinity of the active site where one side of the pocket is formed by loop 7 . Those water molecules are highly suspected to act as nucleophiles. The crystallographic data showed the exiting water molecule of the pocket involved in bridging $\mathbf{E}_{\mathrm{AMY} 2} \mathbf{2 0 4}$ and $\mathbf{D}_{\mathrm{AMY} 2} 289$. One could suspect that the displacement of the loop would position the water molecule as the attacking nucleophile. A further understanding of the AMY2 loop would help to analyze the dynamic behavior of the water molecules during the loop motion. These calculations would give a more realistic energetic pathway for the loop motion with some insight on the actual supply of water molecules as nucleophiles. As loop 7 could be considered as a structural and a functional motif, an ultimate work would be a research of such conserved motifs combined with a dynamic exploration on other amylases of the family 13 .

\section{REFERENCES}

1. Gerstein, M.; Lesk, A. M.; Chotia, C. Biochemistry 1994, 33, 22, 6739-6749.

2. Arteca, A. A. Biopolymers 1996, 39, 671-680.
3. Van Vlijmen, H. W. T.; Karplus, M. J Mol Biol 1997, 267, 975-1001.

4. Brzovic, P. S.; Hyde, C. C.; Miles, E. W.; Dunn, M. F. Biochemistry 1993, 10404-10413.

5. André, G.; Kanchanawong, P.; Palma, R.; Cho, H.; Deng, X.; Irwin, D.; Himmel, M. E.; Wilson, D. B.; Brady J. W. Protein Eng 2003, 16, 125-134.

6. Joseph, D.; Petsko, G. A.; Karplus, M. Science 1990, 249, 1425-1428.

7. Rhee, S.; Parris, K. D.; Hyde, C. C.; Ahmed, S. A.; Miles, E. W.; Davies, D. R, Biochemistry 1997, 36, 7664-7680.

8. McMillan, M. F.; Cahoon, M.; White, A.; Hedstrom, L.; Petsko, G.; Ringe, D, Biochemistry 2000, 39, 45334542.

9. Ring, C. S.; Kneller, D. G.; Langridge, R.; Cohen, F. E. J. Mol. Biol 1992, 224, 685-699.

10. Fetrow, J. S. FASEB J 1995, 9, 708-717.

11. Matsuura, Y.; Kusunoki, M.; Harada, W.; Kakudo, M. J Biochem 1984, 95, 697-702.

12. Brzozowski, A. M.; Davies, G. J. Biochemistry 1997, 36, 10837-10845.

13. Boel, E.; Brady, L.; Brzozowski, A. M.; Derewenda, Z.; Dodson, G. G.; Jensen, V. J.; Petersen, S. B.; Swift, H.; Thim, L.; Woldike, H. F. Biochemistry 1990, 29, 6244-6249.

14. Machius, M.; Wiegand, G.; Huber, R. J Mol Biol 1995, 246, 545-559.

15. Fujimoto, Z.; Takase, K.; Doui, N.; Momma, M.; Matsumoto, T.; Mizuno, H. J Mol Biol 1998, 277, 393407.

16. Aghajari, N.; Feller, G.; Gerday, C.; Haser, R. Protein Sci 1998, 7, 564-572.

17. Strobl, S.; Maskos, K.; Betz, M.; Wiegand, G.; Huber, R.; Gomis-Rüth, F. X.; Glockshuber, R. J Mol Biol 1998, 278, 617-628.

18. Qian, M.; Haser, R.; Payan, F. J Mol Biol 1993, 231, 785-799.

19. Qian, M.; Haser, R.; Buisson, G.; Duée, E.; Payan, F. Biochemistry 1994, 33, 6284-6289.

20. Brayer, G. D.; Luo, Y.;Withers, S. G. Protein Sci 1995, 4, 1730-1742.

21. Ramasubbu, N.; Bhandary, K. K.; Scannapieco, F. A.; Levine, M. J. Proteins Struct Funct Genet 1991, 11, 230-232.

22. Ramasubbu, N.; Paloth, V.; Luo, Y.; Brayer, G. D.; Levine, M. J. Acta Crystallogr 1996, D52, 435-446.

23. Kadziola, A.; Abe, J.; Svensson, B.; Haser, R. J Mol Biol 1994, 239, 104-121.

24. Kadziola, A.; Søgaard, M.; Svensson, B.; Haser, R. J Mol Biol 1998, 278, 205-217.

25. Gilles, C.; Astier, J. P.; Marchis-Mouren, G.; Cambillau, C.; Payan, F. Eur J Biochem 1996, 238, 561-569.

26. Ramasubbu, N.; Ragunath, C.; Mishra, P. J. J Mol Biol 2003, 325, 1061-1076.

27. André, G.; Buléon, A.; Haser, R.; Tran, V. Biopolymers 1999, 50, 751-762. 
28. André, G.; Tran, V. Special Publication No. 246, The Royal Society of Chemistry; Gilbert, H. J., Davies, G. J., Henrissat, B., Svensson, B., Eds.; Cambridge, 1999 pp 165-174.

29. Uitdehaag, J. C. M.; Mosi, R.; Kalk, K. H.; van der Veen, B. A.; Dijkhuizen, L.; Withers, S. G.; Dijkstra, B. Nature Struct Biol 1999, 6, 432-436.

30. Gao, J. In Reviews in Computational Chemistry: Methods and Applications of Combined Quantum Mechanical and Molecular Mechanical Potentials; Lipkowitz, K. B., Boyd, D. B., Eds.; VCH Publishers: New York, 1996.

31. André, G.; Buléon, A.; Juy, M.; Aghajari, N.; Haser, R.; Tran, V. Biopolymers 1999, 49, 107-119.

32. Mouawad, L.; Perahia, D. Biopolymers 1993, 33, 299 611.

33. Mouawad, L.; Perahia, D. J Mol Biol 1996, 258, 393-410.
34. Hayward, S.; Kitao, A.; Berendsen, H. J. C. Proteins Struct Funct Genet 1997, 27, 425-437.

35. Leszczynski, J. F.; Rose, G. D. Science 1986, 234, 849-855.

36. Fetrow, J. S.; Spitzer, J. S.; Gilden, B. M.; Scott, J. M.; Begley, T. J.; Haas, B. J.; Boose, T. Biochemistry 1998, 37, 2477-2487.

37. Quiocho, F. A. Ann Rev. Biochem 1986, 55, 287315.

38. Quiocho, F. A. Pure Appl Chem 1989, 61, 1293-1306.

39. Vyas, N. K. Curr Opin Struct Biol 1991,1, 732-740.

40. Bak-Jensen, K.; André, G.; Tran, V.; Svensson, B. $\mathrm{J}$ Biol Chem, to be published.

Reviewing Editor: Dr. C. Allen Bush 